\title{
EFEITO DE LASERS DE BAIXA POTÊNCIA NO REPARO DE LESÕES CUTÂNEAS
}

\section{EFFECT OF LOW POWER LASERS IN SKIN INJURY REPAIR}

\author{
Camila CARNEIRO ${ }^{1}$, Juliana C. SCHLEDER ${ }^{2}$, Stefani V. FISCHER ${ }^{3 *}$, Rosário A. M. \\ ZEDEBSKI $^{4}$, Fernanda A. VERNER ${ }^{5}$, Leandro LIPINSKI ${ }^{6}$
}

${ }^{1}$ Acadêmica do Curso de Fisioterapia do Centro de Ensino Superior dos Campos Gerais. Ponta Grossa - Paraná / Brasil. E-mail: kmilinha1301@hotmail.com

${ }^{2}$ Doutoranda em Fisiologia Humana, Universidade Federal do Paraná, Curitiba- Paraná/ Brasil. E-mail: juschleder@yahoo.com.br

$3^{3 *}$ Autor para contato: Doutoranda em Fisiologia Humana, Departamento de Fisiologia Humana, Universidade Federal do Paraná - UFPR. Av. Coronel Francisco Heráclito dos Santos, 210 - Jardim das Américas, Curitiba - PR, 81531-970. E-mail: stefaniduda@gmail. com

${ }^{4}$ Profa Dra do Centro de Ensino Superior dos Campos Gerais. Ponta Grossa - Paraná / Brasil. E-mail: rosaario@terra.com.br

${ }^{5}$ Fisioterapeuta do Hospital Universitário Cajuru com especialização em Fisioterapia Oncológica. E-mail: fernanda.verner@hotmail.com

${ }^{6}$ Prof. Dr. do Curso de Medicina Veterinária do Centro de Ensino Superior dos Campos Gerais. Ponta Grossa - Paraná / Brasil. E-mail: leandrolipinski@yahoo.com.br

\section{RESUMO}

Para a redução do tempo de reparo tecidual, a fisioterapia dispõe de recursos, dentre eles o laser. O objetivo deste artigo foi relatar o efeito dos lasers alumínio-gálio-índiofósforo (AlGaInP) e arseniato de gálio ( $\mathrm{AsGa}$ ) como estimuladores no processo de reparo tecidual. A pesquisa foi prospectiva, aplicada e de caráter experimental. A amostra foi constituída por coelhos. Foram induzidas 3 feridas em cada animal, as quais foram divididas em controle e tratamentos com $\mathrm{AlGaInP}$ e com AsGa. O tamanho das feridas foi mensurado em dias alternados até o final do tratamento. Em relação ao tamanho inicial das feridas, o grupo controle apresentou área média de $5,22( \pm 1,79) \mathrm{cm}^{2}$, o grupo AlGaInP apresentou área média de $5,95( \pm 1,79) \mathrm{cm}^{2}$ e o grupo AsGa apresentou área média de $6,67( \pm 1,49) \mathrm{cm}^{2}$. Na verificação da diferença estatística das médias das áreas iniciais entre os grupos, por meio de ANOVA de duas vias, verificou-se que não houve diferença entre os grupos (AlGaInP e controle $-p=0,542$; AsGa e controle $-p=0,125$; AlGaInP e AsGa $-p=0,520)$. Na última avaliação do tamanho das feridas, o grupo controle obteve uma média de $0,33( \pm 0,13) \mathrm{cm}^{2}$, o grupo AlGaInP ficou com média de $0,51( \pm 0,19) \mathrm{cm}^{2}$ e o grupo AsGa apresentou média de $0,36( \pm 0,14) \mathrm{cm}^{2}$. Comparando a primeira e as últimas avaliações por intermédio do teste não paramétrico de Tukey, os três grupos apresentaram diferença estatisticamente significativa $(p=0,0001)$. Não houve diferença no tempo total de cicatrização das feridas tratadas com os lasers AlGaInP e AsGa quando comparadas ao grupo controle.

Palavras-chave: Cicatrização. Lasers. Modalidades de fisioterapia.

\begin{abstract}
To reduce the time of wound healing, physiotherapy has some resources, including the laser. The aim of this paper was to report the effect of lasers aluminum-galliumindium-phosphorus (AlGaInP) and gallium arsenide (GaAs) as stimulators in tissue repair process. This was a prospective, applied and experimental study. The sample
\end{abstract}


consisted of rabbits. Three wounds were induced in each animal, which were divided into control and treatments AlGaInP, and treatment with GaAs. The wound area was measured every two days until the end of treatment. Compared to the initial size of the wounds, the control group showed a mean area of $5.22( \pm 1.79) \mathrm{cm}^{2}$, AlGaInP showed an average area of $5.95( \pm 1.79) \mathrm{cm}^{2}$ and AsGa an average area of $6.67( \pm 1.49) \mathrm{cm}^{2}$. When verifying the statistical difference of the average of the initial areas among the groups through two-way ANOVA, it was observed that there was no difference among the groups (AlGaInP and control $\mathrm{p}=0.542$; GaAs and control $\mathrm{p}=0.125$; $\mathrm{AlGaInP}$ and GaAs $\mathrm{p}=0.520$ ). In the last assessment of the size of the wounds, the control group had an average of $0.33( \pm 0.13) \mathrm{cm}^{2}$, the AlGaInP group had an average of $0.51( \pm 0.19)$ $\mathrm{cm}^{2}$ and the GaAs group had $0.36( \pm 0.14) \mathrm{cm}^{2}$. When comparing the first and last assessment through the nonparametric Tukey test, the three groups showed statistically significant difference $(\mathrm{p}=0.0001)$. There was no difference in healing time of wounds treated with GaAs and AlGaInP lasers when compared to the control group

Keywords: Wound healing. Laser therapy. Physiotherapy.

\section{INTRODUÇÃO}

Os tecidos, quando lesados, iniciam o processo de reparo composto por três fases: inflamatória, proliferativa e maturação. O processo de reparo recupera as estruturas celulares e os tecidos que foram danificados (BALBINO; PEREIRA; CURI, 2003). Alguns fatores beneficiam a cicatrização de feridas, acelerando o processo e diminuindo o tempo da fase inflamatória, de proliferação celular e de remodelamento do tecido (BUSNARDO; BIONDO-SIMÕES, 2010).

A laserterapia apresenta efeito estimulante no processo de reparo tecidual, agindo sobre os fotorreceptores que são de grande importância na cadeia respiratória das mitocôndrias, aumentando a produção de adenosina trifosfato, aumenta a velocidade mitótica das células, e, consequentemente, aceleração no processo celular de reparo (MOURA; SILVA; GODOY, 2005).

A fisioterapia dispõe de vários recursos que visam melhorar o bem-estar físico e psíquico do paciente. Dentre os recursos fisioterapêuticos aplicados em fase de recuperação de tecidos lesados, destaca-se o laser de baixa potência. Esse recurso atua em nível celular, por meio da estimulação fotoquímica, podendo, assim, promover o aumento do metabolismo celular (FERREIRA, 2006). Atribuem-se ao laser os efeitos analgésico, antiedematoso, anti-inflamatório e de reparação tecidual (TATARUNAS; MATERA; DAGLI, 1998; RODRIGUES; MAIOR; AQUINO, 2009; GONÇALVES et al., 2010).

Dentre os vários tipos de lasers de baixa potência estão: alumínio-gálio-índio-fósforo (AlGaInP), gálio-alumínio-arseneto (GaAlAs), arseniato de gálio (AsGa) e o hélio neônio (HeNe) (KITCHEN, 2010). Na prática fisioterapêutica, os lasers AlGaInP e AsGa são os mais frequentemente utilizados. O laser AlGaInP apresenta comprimento de onda de $670 \mathrm{~nm}$ com luz vermelha visível, apresentando maior eficácia em lesões como feridas, úlceras e queimaduras (BUSNARDO; BIONDO-SIMÕES, 2010), com emissão de onda de forma pulsada (AGNE, 2004; KITCHEN, 2010). O laser AsGa tem comprimento de onda de $904 \mathrm{~nm}$, apresentando luz invisível, mais utilizado em lesões como doença articular, muscular e tendinosa, com emissão de onda de forma contínua, como demonstram alguns estudos (SILVEIRA, 2009).

Alguns estudos (STEFANELLO; HAMERSKI, 2006; NAYAK; MAIYA; KUMAR, 2007; COSTARDI, 2008; SILVEIRA, 2009) mostram a utilização de lasers AlGaInP (NASCIMENTO, 2006; BUSNARDO; BIONDO-SIMÕES, 2010) e AsGa (PINTO et al., 2008; GONÇALVES et al., 2010) na otimização do processo cicatricial de tecidos. No entanto, ainda existem lacunas no meio científico sobre a melhor dose, tempo e comprimento de onda. Na prática clínica, a escolha de tratamentos adequados acelera o processo de reparo tecidual.

O presente estudo teve por objetivo comparar e verificar o efeito da laserterapia com AlGaInP e AsGa no tempo de cicatrização de feridas induzidas em dorso de coelhos. 


\section{MATERIAL E MÉTODO}

Pesquisa prospectiva, aplicada de caráter experimental, realizada de forma quantitativa (MARCONI; LAKATOS, 2010). Foi aprovada pelo Comitê de Ética em Pesquisa do Centro de Ensino Superior dos Campos Gerais em Experimentação Animal, sob o número de registro 704/ SCEPA. A amostra foi composta por sete coelhos da raça Nova Zelândia, de origem controlada, apresentando idade entre 120 a 160 dias, mantidos em gaiolas individuais $-50 \mathrm{~cm}$ por $35 \mathrm{~cm}$ - forradas com cepilho, fotoperíodo de 12 horas, temperatura ambiente $26^{\circ} \mathrm{C}$ e ração comercial ad libidum.

\section{Indução da lesão}

Após jejum alimentar (12 horas), os animais foram tranquilizados com cloridrato de xilazina $0,04 \mathrm{mg} /$ $\mathrm{kg}$ (i.m), posteriormente tricotomizados, utilizando-se um navalhete de barbear ProArt ${ }^{\circledR}$. Antes da incisão, aplicou-se cloridrato de lidocaína nos locais. Com auxílio de um bisturi, foram realizadas três lesões cutâneas no dorso direito de cada animal. Cada lesão media $2 \mathrm{~cm}$ por $2 \mathrm{~cm}$ e o procedimento foi realizado sem antissepsia. As lesões foram feitas a $2 \mathrm{~cm}$ da coluna vertebral e respeitando a distância de $2 \mathrm{~cm}$ entre cada lesão. Cada animal apresentava três lesões: uma foi tratada com AlGaInP, a outra com AsGa e a última não foi tratada (controle), para que o resultado fosse fidedigno ao tratamento e não resultante das diferenças individuais de cada animal.

\section{Tratamento}

O tratamento foi iniciado 12 horas após a indução das lesões, havendo dois dias de intervalo entre cada avaliação, durante 20 dias. $O$ grupo controle permaneceu sem tratamento. Para ambos os grupos tratados com laser AlGaInP e AlGaInP, as aplicações foram realizadas de forma pontual, dose de $4 \mathrm{~J} / \mathrm{cm}^{2}$ (SILVEIRA, 2009) ao redor da lesão, sem contato direto (distância aproximada de um milímetro entre a ponta da caneta e a lesão). Caneta foi mantida perpendicularmente à lesão com espaço entre os pontos de $1 \mathrm{~cm}$.

\section{Avaliações}

As avaliações foram realizadas até o $20^{\circ}$ dia após incisão, havendo intervalo de dois dias entre as avaliações. A mensuração da área de cada ferida foi realizada, colocando-se um papel de transparência sobre a ferida e demarcando com caneta de retroprojetor de ponta média a sua circunferência, para avaliação da retração. Após a obtenção da circunferência ou elipse, no final de cada aplicação, foi realizada a medição com o compasso algébrico, obtendo-se uma medida fiel do raio da circunferência ou medidas da elipse, a fim de se obter a área das feridas. Após o término do estudo, os coelhos foram eutanasiados com dose extra de anestésico.

\section{Análise estatística}

Todos os resultados foram apresentados com valores da média \pm erro padrão da média (SEM). Para análise estatística, foi utilizado o programa estatístico GraphPad Prism 6. Testes e pós-testes aplicados seguem descritos nas respectivas legendas. A diferença foi considerada significativa quando o valor de $p$ foi igual ou inferior a $0,05(p \leq 0,05)$.

\section{RESULTADOS}

As médias das áreas das feridas na primeira avaliação foram comparadas entre os grupos experimentais: Controle versus $\mathrm{AlGaInP}$ com valor $p=0,542$; Controle versus AsGa valor $p=0,125$; e AlGaInP versus AsGa valor $p=0,520$. Com base nos valores obtidos, observou-se que, inicialmente, não houve diferença significativa entre os tamanhos das feridas nos grupos analisados.

Evolução da área das feridas para os grupos Controle, AlGaInP e AsGa ao longo de 10 avaliações (Figuras 1, 2 e 3, respectivamente).

Figura 1 - Evolução da área das feridas do Grupo Controle. Grupo Controle

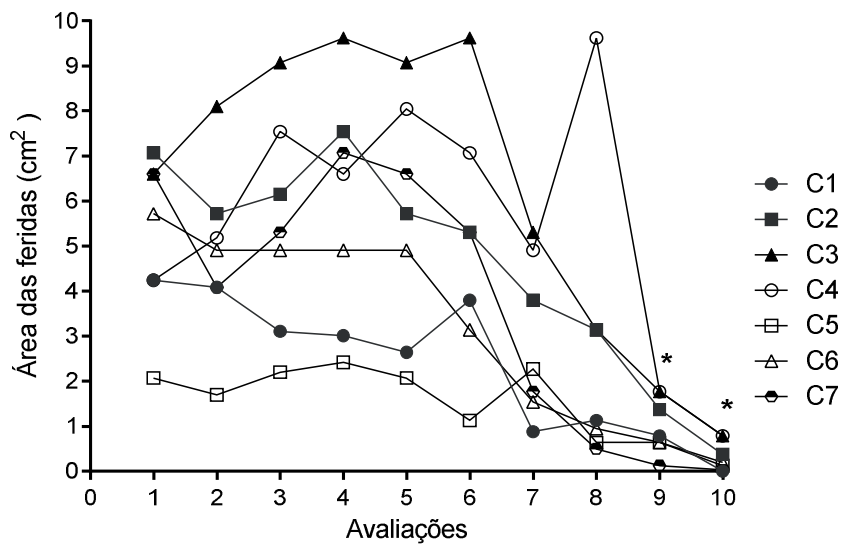

As médias das avaliações foram comparadas à média do primeiro dia de tratamento. Valores expressos média \pm SEM. O símbolo “*” representa a diferença estatística referente ao teste ANOVA de duas vias, pós-teste de Tukey. As letras "C" representam cada animal $(n=7)$. 
Figura 2 - Evolução da área das feridas do Grupo AlGaInP. Grupo AIGalnP

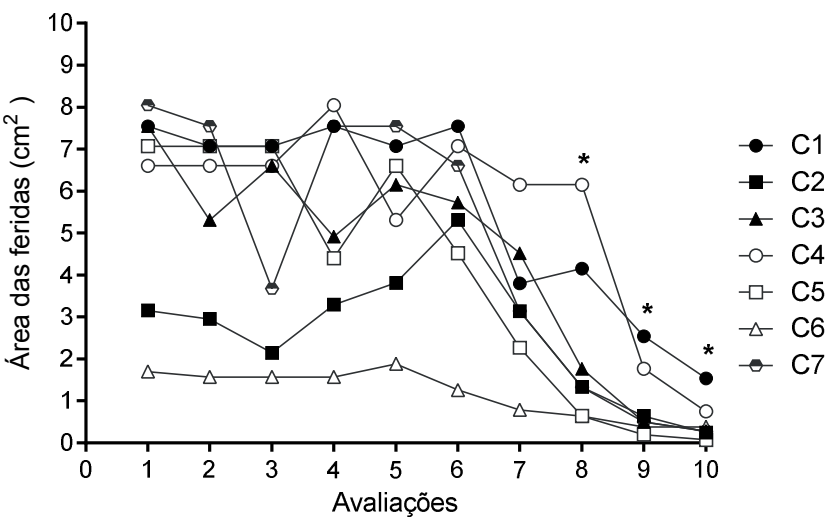

As médias das avaliações foram comparadas à média do primeiro dia de tratamento. Valores expressos média \pm SEM. O símbolo "*” representa a diferença estatística referente ao teste ANOVA de duas vias, pós-teste de Tukey. As letras "C" representam cada animal $(n=7)$.
Figura 3 - Evolução da área das feridas do Grupo AsGa Grupo AsGa

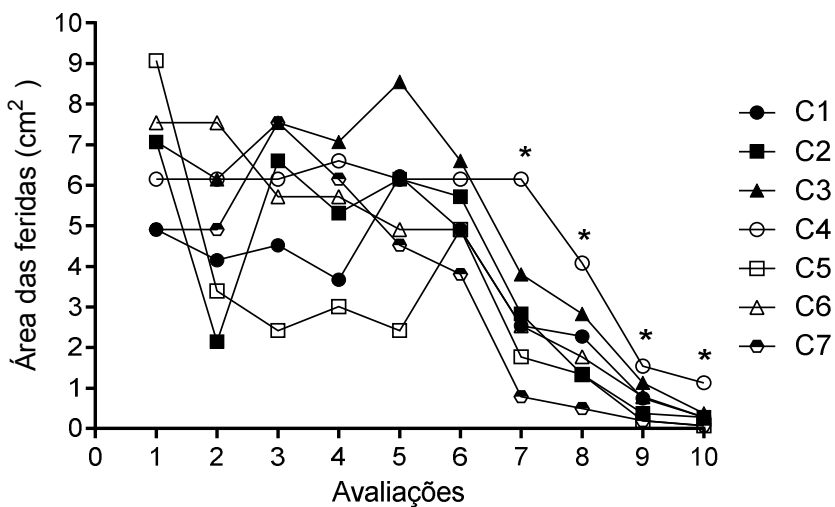

As médias das avaliações foram comparadas à média do primeiro dia de tratamento. Valores expressos média \pm SEM. O símbolo “*” representa a diferença estatística referente ao teste ANOVA de duas vias, pós-teste de Tukey. As letras "C" representam cada animal $(n=7)$.

Na Tabela 1, apresentamos a evolução das áreas das feridas dos coelhos durante as avaliações.

Tabela 1 - Evolução das áreas das feridas com diferentes tratamentos

\begin{tabular}{|c|c|c|c|c|c|c|c|c|c|c|c|}
\hline \multicolumn{12}{|c|}{ Área das feridas $\left(\mathrm{cm}^{2}\right)$} \\
\hline \multicolumn{2}{|c|}{ Avaliação } & 1 & 2 & 3 & 4 & 5 & 6 & 7 & 8 & 9 & 10 \\
\hline \multirow{3}{*}{$\begin{array}{l}\overrightarrow{0} \\
\frac{\bar{\sigma}}{\overline{0}} \\
0\end{array}$} & Controle & 4,24 & 4,08 & 3,11 & 3,01 & 2,64 & 3,8 & 0,88 & 1,13 & 0,79 & 0 \\
\hline & AlGaInP & 7,54 & 7,07 & 7,07 & 7,54 & 7,07 & 7,54 & 3,8 & 4,15 & 2,54 & 1,54 \\
\hline & AsGa & 4,91 & 4,15 & 4,52 & 3,67 & 6,22 & 4,91 & 2,54 & 2,27 & 0,75 & 0,28 \\
\hline \multirow{3}{*}{ 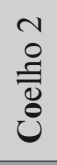 } & Controle & 7,07 & 5,72 & 6,15 & 7,54 & 5,72 & 5,31 & 3,8 & 3,14 & 1,37 & 0,38 \\
\hline & AlGaInP & 3,15 & 2,95 & 2,15 & 3,29 & 3,81 & 5,31 & 3,14 & 1,33 & 0,64 & 0,25 \\
\hline & AsGa & 7,07 & 2,15 & 6,6 & 5,31 & 6,15 & 5,72 & 2,83 & 1,33 & 0,38 & 0,28 \\
\hline \multirow{3}{*}{$\begin{array}{l}m \\
\text { o } \\
\overline{0} \\
\dot{0} \\
\dot{0}\end{array}$} & Controle & 6,6 & 8,1 & 9,07 & 9,62 & 9,07 & 9,62 & 5,31 & 3,14 & 1,77 & 0,79 \\
\hline & AlGaInP & 7,54 & 5,31 & 6,6 & 4,91 & 6,15 & 5,72 & 4,52 & 1,77 & 0,5 & 0,28 \\
\hline & AsGa & 7,07 & 6,15 & 7,54 & 7,07 & 8,55 & 6,6 & 3,8 & 2,83 & 1,13 & 0,38 \\
\hline \multirow{3}{*}{$\begin{array}{l}+ \\
\text { ஓ } \\
\stackrel{\Xi}{0} \\
\dot{\theta}\end{array}$} & Controle & 4,24 & 5,18 & 7,54 & 6,6 & 8,04 & 7,07 & 4,91 & 9,62 & 1,77 & 0,79 \\
\hline & AlGaInP & 6,6 & 6,6 & 6,6 & 8,04 & 5,31 & 7,07 & 6,15 & 6,15 & 1,77 & 0,75 \\
\hline & AsGa & 6,15 & 6,15 & 6,15 & 6,6 & 6,15 & 6,15 & 6,15 & 4,08 & 1,54 & 1,13 \\
\hline \multirow{3}{*}{$\begin{array}{l}n \\
\text { in } \\
\overline{0} \\
\dot{0}\end{array}$} & Controle & 2,07 & 1,7 & 2,2 & 2,42 & 2,07 & 1,13 & 2,27 & 0,64 & 0,64 & 0,13 \\
\hline & AlGaInP & 7,07 & 7,07 & 7,07 & 4,4 & 6,6 & 4,52 & 2,27 & 0,64 & 0,2 & 0,07 \\
\hline & AsGa & 9,07 & 3,39 & 2,42 & 3,01 & 2,42 & 4,91 & 1,77 & 1,33 & 0,2 & 0,07 \\
\hline \multirow{3}{*}{$\begin{array}{l}0 \\
\circ \\
\stackrel{\overline{0}}{0} \\
\dot{0}\end{array}$} & Controle & 5,72 & 4,91 & 4,91 & 4,91 & 4,91 & 3,14 & 1,54 & 0,95 & 0,64 & 0,2 \\
\hline & AlGaInP & 1,7 & 1,57 & 1,57 & 1,57 & 1,88 & 1,26 & 0,79 & 0,64 & 0,38 & 0,38 \\
\hline & AsGa & 7,54 & 7,54 & 5,72 & 5,72 & 4,91 & 4,91 & 2,54 & 1,77 & 0,79 & 0,28 \\
\hline \multirow{3}{*}{$\begin{array}{l}\hat{0} \\
\text { o } \\
\overline{0} \\
\dot{\theta}\end{array}$} & Controle & 6,6 & 4,08 & 5,31 & 7,07 & 6,6 & 5,31 & 1,77 & 0,5 & 0,13 & 0,03 \\
\hline & AlGaInP & 8,04 & 7,54 & 3,67 & 7,54 & 7,54 & 6,6 & 3,14 & 1,33 & 0,5 & 0,28 \\
\hline & AsGa & 4,91 & 4,91 & 7,54 & 6,15 & 4,52 & 3,8 & 0,79 & 0,5 & 0,2 & 0,07 \\
\hline
\end{tabular}


As médias das áreas das feridas do grupo controle na primeira e última avaliação foram 5,22 $\pm 0,68$ $\mathrm{cm}^{2}$ e $0,33 \pm 0,13 \mathrm{~cm}^{2}$, respectivamente. Na comparação dessas avaliações, obteve-se valor $\mathrm{p}=0,0001$. $\mathrm{O}$ grupo AsGa, na primeira avaliação, obteve média de $6,67 \pm 0,57 \mathrm{~cm}^{2}$ e na última $0,36 \pm 0,14 \mathrm{~cm}^{2}$, com valor $\mathrm{p}=0,0001$. A média das áreas das feridas do grupo AlGaInP foi, na primeira avaliação, $5,95 \pm 0,93 \mathrm{~cm}^{2} \mathrm{e}$, na última avaliação, $0,51 \pm 0,19 \mathrm{~cm}^{2}$, e valor $\mathrm{p}=0,0001$.

A comparação das médias finais das áreas das feridas entre os três grupos mostrou os seguintes valores de p: grupo Controle versus AlGaInP $\mathrm{p}=0,443$, Controle versus AsGa $\mathrm{p}=0,879$, e AlGaInP versus AsGa $p=0,527$. Com bases nesses valores, verificou-se que não houve diferença estatisticamente significativa entre o desfecho dos grupos.

\section{DISCUSSÃO}

A cicatrização é dividida em fase inflamatória, fase de granulação e de remodelagem (BALBINO; PEREIRA; CURI, 2003). Nas divisões das fases da cicatrização, existe complexa e coordenada série de eventos que incluem quimiotaxia, fagocitose, neoformação, degradação e remodelação do colágeno. Além disso, angiogênese, epitelização e produção de novos glicosaminoglicanos e proteoglicanos, os quais são vitais para o meio onde ocorre a cicatrização. Esses processos biológicos resultam, finalmente, na substituição dos tecidos lesados por tecido cicatricial (GARROS; CAMPOS; TÂMBARA, 2006).

A laserterapia, aplicada para estimulação dos processos biológicos da cicatrização, é amplamente utilizada em vários estudos com modelos animais, induzindo a aceleração da cicatrização da ferida (BUSNARDO; BIONDO-SIMÕES, 2010; MELO, 2011). Neste estudo clínico, em decorrência da mobilidade do tecido seccionado da amostra, algumas feridas, após sua incisão, apresentaram-se maiores em relação ao tamanho de $2 \mathrm{~cm}^{2} \times 2 \mathrm{~cm}^{2}$ proposto (RODRIGUES; MAIOR; AQUINO, 2009). Segundo Cross (1995), isso ocorre dada a tensão elástica da pele circunjacente, perda da aderência à fáscia profunda e mobilidade da pele, nesse modelo experimental.

Quando analisadas na fase inicial, que representa a fase inflamatória, houve expansão da área da lesão, bordas edemaciadas e hiperemia, tanto nos grupos tratados com os lasers, como no grupo controle. Tais mudanças foram observadas visualmente, mas não foram quantificadas. No trabalho de Tatarunas, Matera e Dagli (1998), realizado com gatos, todos realizaram ovariohisterectomia e foram divididos em 3 grupos, aleatoriamente: Grupo A: recebeu radiação laser única de $4 \mathrm{~J} / \mathrm{cm}^{2}$; Grupo B: radiação laser única de $2 \mathrm{~J} / \mathrm{cm}^{2}$; e Grupo C: controle, não foram radiados. No segundo dia, foi observado tecido conjuntivo subjacente com edema e congestão com infiltrado inflamatório composto predominantemente por neutrófilos íntegros e degenerados. No grupo B, na derme e na hipoderme, infiltrado inflamatório com predomínio de polimorfonucleares e marginação leucocitária. No grupo C, epitélio se regenerando, infiltrado inflamatório em derme e intensa congestão com predomínio de leucócitos polimorfonucleares.

Neste estudo, ao observar-se o grupo controle, a partir da nona avaliação, os animais tiveram um declínio na área das feridas (Tabela 1). Acredita-se que o grupo apresentou uma diminuição da área da ferida devido ao término da fase inflamatória e início da fase de proliferação que é responsável pelo fechamento da ferida em si (MENDONÇA; COUTINHO-NETTO, 2009). Apenas o espécime $C 4$ apresentou um aumento desse campo no oitavo dia da avaliação, o qual demonstrou uma exacerbação da fase inflamatória, apresentando exsudato na lesão, sem demonstrar melhora da ferida desde o início da avaliação macroscópica. Destacamos o exemplar para demonstrar que, mesmo sob as mesmas condições de ambiente e tratamento, os organismos têm comportamento próprio, podendo reagir de forma diferenciada dos demais espécimes do grupo.

A área das feridas do grupo AlGaInP apresentou queda do tamanho após o oitavo dia de avaliação, não apresentando diferença entre este e o grupo controle. A ferida tratada com o AlGaInP mostrou menor área no $10^{\circ}$ dia de avaliação (Tabela 1). Diferentemente do estudo realizado por Nayak, Maiya e Kumar (2007), realizado com modelo experimental murino (Winstar), dividido em dois grupos - controle e AlGaInP - que demonstrou uma diferença significativa na redução da área da ferida tratada com hélio neônio. Contudo, não foi esclarecido em qual período do estudo isso foi verificado. Neste estudo, o espécime $\mathrm{C} 1$ apresentou uma redução maior na ferida tratada com AlGaInP. Acredita-se que o resultado foi proporcional à área inicial de ferida. No grupo tratado com o laser AsGa, 
em sua maioria, as feridas apresentaram redução da área a partir do sétimo dia de avaliação.

Como observado, a cicatrização apresentou-se progressiva para os três grupos experimentais. Comparando as médias das áreas das feridas dentro do próprio grupo, a cada avaliação com o dia um de tratamento, observamos que os grupos com laserterapia tenderam a reduzir a área da ferida antes que o grupo controle. No entanto, não houve diferenças quanto ao tempo no fechamento das lesões quando comparados os três grupos experimentais. O mesmo foi observado em estudo de Beheregaray et al. (2010). Os autores citam a melhora em aspectos como proliferação vascular, colagenização e crescimento de folículos pilosos. Bish et al. (1999) ainda citam os efeitos bioestimulátorios da apliacação da laserterapia, apresentando efeitos sistêmicos, os quais podem influenciar as lesões próximas sem aplicação de laser, podendo mascarar os efeitos terapêuticos do laser quando comparado à lesão controle contralateral.

Os resultados obtidos no presente estudo não anuem os resultados dos estudos citados nas referências. Conscientes de que apenas os aspectos clínicos não sejam suficientes para conclusão, acredita-se na necessidade de um estudo microscópico, avaliando a morfometria do tecido cicatricial bem como os efeitos sistêmicos da laserterapia, concomitantes, para confirmação dos resultados.

\section{CONCLUSÃO}

No ensaio clínico realizado, houve uma redução significativa do tamanho da área das feridas nos três grupos. Não houve diferença significativa no tempo de cicatrização total da ferida. No entanto, os grupos com laserterapia iniciaram a redução na área da ferida antes do grupo controle. A partir dessa constatação, sugere-se a execução de novos trabalhos, com critérios de avaliação mais precisos, estendendo-se o período de avaliação até a remodelação natural do colágeno, com a análise microscópica simultânea.

\section{REFERÊNCIAS}

AGNE, J. Eletrotermoterapia Teoria e Prática. Santa Maria: Orium. 2004.

BALBINO, C. A. ; PEREIRA, L. M.; CURI, R. Mecanismos envolvidos na cicatrização: uma revisão. Revista Brasileira de Ciências Farmacêuticas. Ilhéus, 41 (1) :27-51, 2005.
BEHEREGARAY, W. K.; GIANOTTI, G. C.; LEAL, J. S. et al. Uso do laser ALGaInP na cicatrização de lesões cutâneas experimentais em coelhos. Acta Scientiae Veterinariae. Porto Alegre. 38(3): 237-243, 2010.

BISHT, D.; MEHROTRA, R.; SINGH, P. A. et al. Effect of helium-neon laser on wound healing. The Journal of Experimental Biology. Indian. 37 (2):187-9, 1999.

BUSNARDO, V. L.; BIONDO-SIMÕES, M. L. Effects of low-level helium-neon laser on induced wound healing in rats. Revista Brasileira de Fisioterapia. São Carlos. 14 (1) :45-51, 2010.

COSTARDI, C. H. Z. Efeito do laser de baixa intensidade $(670 \mathrm{~nm})$ após contusão muscular em ratos. Fisioterapia e Movimento. São Paulo. 21 (2) :21-30, 2008.

CROSS, S. E.; NAYLOR, I. L.; COLEMAN, R. A. et al. An experimental model to investigate the dynamics of wound contraction. British Journal of Plastic Surgery. Hertfordshire. 48 (4) :189-197, 1995.

FERREIRA, M. A. Efeitos do laser de baixo intensidade no processo de cicatrização em ratos jovens e idosos: Estudo morfométrico e morfológico. Alfeneras, MG, 2006. 61f. Dissertação (Mestrado em Ciências da Saúde) - Programa de Pós-Graduação em Ciências da Saúde, Universidade José do Rosário Vellano.

GARROS, I. C.; CAMPOS, A. C. L.; TÂMBARA, E. M. Extrato de Passiflora edulis na cicatrização de feridas cutâneas abertas em ratos: Estudo Morfológico e Histológico. Acta Cirúrgica Brasileira, Maranhão. 21 (3) 55-64, 2006.

GONÇALVES, R. V, et al. Influência do laser arseneto de gálio-alumínio em feridas cutâneas de ratos. Revista Fisioterapia e Movimento. Curitiba, 23 (3):381-388, 2010.

KITCHEN, S. B. S. Eletroterapia. $11^{\circ}$ edição São Paulo, Manole. 2010.

MARCONI, M. A.; LAKATOS, E. M. Metodologia do Trabalho Científico. 6. ed. São Paulo: Atlas. 2010.

MELO, V. A. Effect of low level laser on sutured wound healing in rats. Acta Cirúrgica Brasileira. São Paulo, 26 (2), 2011.

MENDONÇA, R. J.; COUTINHO-NETTO, J. Aspectos celulares da cicatrização. Anais Brasileiros de Dermatologia. Rio de Janeiro, 84 (3) :257-262, 2009.

MOURA, C. E. M.; SILVA, L. L. M.; GODOY, J. R. Úlceras de Pressão: prevenção e tratamento. Univ. Ci. Saúde. (2) :275-286, 2005.

NASCIMENTO, D. G. Efeitos da irradiação com o laser HeNe $632.8 \mathrm{~nm}$ sobre a cicatrização de feridas em ratos. Ciência, Cuidado e Saúde. Maringá, 5 (2) :229-235, 2006. 
NAYAK, B. S.; MAIYA, A.; KUMAR, P. Influence of Helium-Neon Laser Photostimulation on Excision. Online Journal Of Biological Sciences, St. Augustine, Trinidad. 8992, 2007.

PINTO, M. V. M., et al. Comparative study of the effects of the Ga-As $(904 \mathrm{~nm}, 150 \mathrm{~mW})$ laser and the pulsed ultrasound of $1 \mathrm{MHz}$ in inflammation of tibialis muscle of Wistar rats. Brazilian Archives of Biology and Technology. Curitiba, 51, 2008.

RODRIGUES, S. S. M. F. G., MAIOR, B.S.S., AQUINO, D.R., et al. Efeitos do laser de baixa potência, sob diferentes protocolos, no reparo de feridas em ratos. Clínica e Pesquisa em Odontologia- Unitau. 1 (1) :31-37, 2009.

SILVEIRA, P. C. L. Efeito da Laserterapia de baixa potência na resposta oxidativa epidérmica induzida pela cicatrização de feridas. Revista Brasileira de Fisioterapia. São Carlos, 13 (4) :281-287, 2009.

STEFANELLO, T. D.; HAMERSKI, C. R. Tratamento de úlcera de pressão através do laser AsGa de $904 \mathrm{~nm}$. Arq. Ciência Saúde Unipar. Umuarama, 10 (2) :99-103, 2006.

TATARUNAS, A. C.; MATERA, J. M.; DAGLI, M. L. Z. Estudo clínico e anatomopatológico da cicatrização cutânea no gato doméstico. Utilização do laser de baixa potência GaAs (904 nm). Acta Cirurgica Brasileira. São Paulo, 13 (2). 1998. 